\title{
Dapsone-induced optic atrophy: a rare case report
}

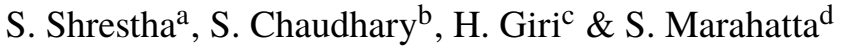 \\ a Junior Resident, Department of Dermatology and Venereology, B.P.Koirala \\ Institute of Health Sciences, Dharan, Nepal \\ ${ }^{\mathrm{b}}$ Assistant Professor, Department of Ophthalmology, B.P.Koirala Institute of \\ Health Sciences, Dharan, Nepal \\ c Junior Resident, Department of Ophthalmology, B.P.Koirala Institute of \\ Health Sciences, Dharan, Nepal \\ ${ }^{\mathrm{d}}$ Associate Professor, Department of Dermatology and Venereology, \\ B.P.Koirala Institute of Health Sciences, Dharan, Nepal
}

Submitted 18 August 2020; Accepted 20 August 2020

\begin{abstract}
Summary Dapsone-induced optic atrophy $(\mathrm{OA})$ is very rare at therapeutic doses. A 43-year-old gentleman on WHO-recommended multibacillary Multi-Drug Therapy (WHO MB-MDT) containing rifampicin, dapsone, and clofazimine, presented with bilateral gradual painless diminution of vision after 3 months of medication. He denied any history of trauma, headache, limb weakness, ataxia, diplopia, temporal pain , or weight loss. There was no history of sudden diminution of vision in family members. Fundus examination showed bilateral pale optic discs with well-defined margins. Glucose-6-phosphate dehydrogenase (G6PD) was normal. He was diagnosed with OA secondary to dapsone. There is therefore a possibility of dapsone induced OA even at the therapeutic dose. Hence, we should perform routine eye check-up before starting dapsone.
\end{abstract}

Keywords: Dapsone, optic atrophy, leprosy

\section{Introduction}

Dapsone has anti-microbial (anti-mycobacterial and anti-protozoal) and anti-inflammatory effects. It is used in the treatment of leprosy, prophylaxis of Pneumocystic jirovecii and toxoplasmosis in AIDS patients, various chronic inflammatory dermatoses and immunebullous disorders. ${ }^{1,2}$ Dapsone has no direct toxic effect on the retina at therapeutic doses, nor in case of overdose. However, there are a few reports of dapsone-induced optic neuritis, optic atrophy (OA) and retinal necrosis. There are six case reports of OA following dapsone overdose $^{3-9}$ and one following therapeutic dose. ${ }^{10}$ In cases of dapsone overdose, OA was acute

Correspondence to: Dr Suchana Marahatta, Associate Professor, Department of Dermatology and Venereology, B.P.Koirala Institute of Health Sciences, Dharan, Nepal (Tel.: +977-9862023236; e-mail: dermasuchana@ gmail.com; suchanamarahatta@yahoo.com) 
in onset, occurring within 1 to 2 weeks. In the single case report of OA following a therapeutic dose of dapsone, the manifestation was reported after one month of therapy. We report an interesting case of OA following administration of a therapeutic dose of dapsone.

\section{Case report}

A 43-year-old gentleman was diagnosed with Borderline Tuberculoid leprosy with normal baseline eye examination. He was started on WHO recommended multibacillary multi-drug therapy for leprosy containing 100mg dapsone daily along with rifampicin and clofazimine. Before starting treatment, a thorough ophthalmic examination was done. The best corrected visual acuity was $6 / 6$ in both the eyes. Corneal sensation was intact, intraocular pressure was normal $(14.2 \mathrm{mmHg}$ in the right eye and $14.6 \mathrm{mmHg}$ in the left eye) and fundus evaluation was within normal limits. After 3 months of treatment, he presented with bilateral sudden onset, progressive diminution of vision. He had controlled diabetes mellitus (DM) and hypertension (HTN) under medication (500mg Metformin and 10mg Amlodipine once daily) for 6 months. He denied intake of large dose of dapsone. He did not give a history of trauma, headache, limb weakness, ataxia, diplopia, temporal pain or weight loss. There was no history of smoking , alcohol consumption or use of other medications besides the above mentioned medications. Family members did not have any history of sudden diminution of vision.

On examination, the patient was well-oriented to time, place and person. There was no pallor, icterus, lymphadenopathy, clubbing, cyanosis or edema. His vitals were stable. On ophthalmic examination, the visual acuity was $1 / 60$ and 3/60 in right and left eye, respectively. The patient could not read all the plates while assessing color vision using the Ishihara pseudo-chromatic chart. The eyes were aligned with no conjunctival congestion, clear corneas, normal anterior chamber depth and clear lenses. Intraocular pressure was comparable to baseline. Fundus examination after pupillary dilatation showed pale optic discs with welldefined margins in both eyes (Figure 1). There were no intraretinal hemorrhages, macular edema or retinal detachment. Blood tests revealed normal complete blood count, liver function tests, blood sugar (fasting and post-prandial), lactate dehydrogenase and glucose-6-phosphate dehydrogenase (G6PD). Blood concentration of dapsone was not done due to unavailability. The patient was informed about the disease, its cause and management. Since the patient had already developed OA, no added intervention was done for this. However, the patient was kept under regular follow-up and ophthalmic check-up. Dapsone was withheld from his regular MBMDT regimen and he is only on rifampicin and clofazimine for leprosy management since last 6 months. ${ }^{11,12}$ Until 6 months of follow-up, his vision and optic atrophy remained static.

\section{Discussion}

Dapsone is a synthetic sulfone that has anti-microbial and anti-inflammatory effects. It is used for various dermatological conditions, including leprosy (in combination with rifampicin and clofazimine in WHO MB-MDT), dermatitis herpetiformis, IgA pemphigus, linear $\operatorname{IgA}$ dermatosis, bullous pemphigoid, and various neutrophilic dermatoses. Besides these, dapsone is also used for various other non-dermatological conditions. Dapsone is associated with various side effects, including hemolytic anemia, methemoglobin formation, photo-sensitivity, anorexia, abdominal pain, nausea, vomiting and hypersensitivity reaction. ${ }^{2}$ There are a few case reports of ophthalmic side effects of dapsone, such as macular damage due to ischemia of the capillaries. 


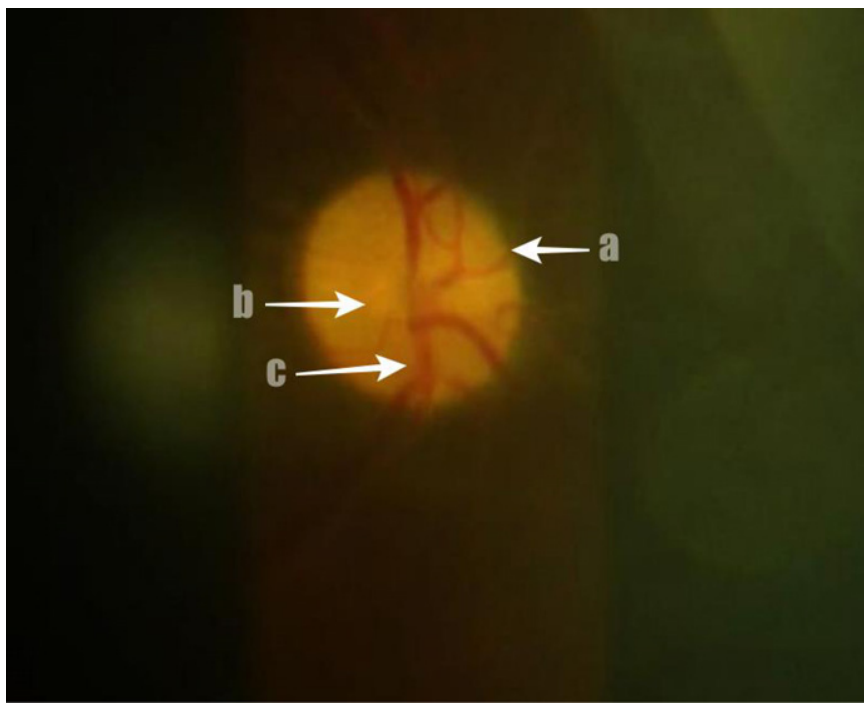

Figure 1 Fundus photography taken from 90 diopter lens under slit lamp showing well-defined optic disc margin (a), pale disc with obliteration of cup disc ratio (b) and attenuated vessels (c).

It has been shown that intake of dapsone even at therapeutic doses can result in fragmentation of red blood cells (RBCs). It has been hypothesized that as retinal capillaries have a small caliber with considerable length, those fragmented RBCs can block them, resulting in ischemic macular damage. However, the exact mechanism is not yet clear. ${ }^{13,14}$

After a thorough history and clinical evaluation, dapsone was the most probable explanation for optic atrophy in our case. However, HTN and DM might have added the risk of optic neuropathy. ${ }^{15,16}$ The fundus examination did not reveal changes pertinent to HTN or DM in our patient. Likewise, simultaneous involvement of both optic nerves is less likely in HTN and DM. ${ }^{17}$ The patient was under regular follow-up at the interval of 1 month and leprosy mediations were provided from the hospital leprosy clinic in a controlled fashion; so, the chance of dapsone overdose is low. Based on these clinical clues and a few published reports, we made the final diagnosis of 'Dapsone-induced Optic Atrophy'. However, we could not support it further with fluorescein angiography because of its unavailability at our center.

On extensive literature search, we could find only one cross-sectional study on causes of OA from Nepal. The study concluded that glaucoma was the most common cause. Other less frequent causes were trauma, vascular events, intracranial space occupying lesions, papilledema, optic neuritis, alcohol intoxication, myopia, age related macular degeneration and idiopathic. ${ }^{18}$

There are only a few case reports of dapsone-induced OA and detailed evidence concerning its mechanism is lacking. One study done in 1982 with 7 patients taking therapeutic doses of dapsone with features of hemolysis, evaluated the optic side effects of dapsone, but there was little evidence of ocular damage caused by therapeutic doses of dapsone. ${ }^{19}$ However, we found a single published case report on dapsone-induced OA at therapeutic doses. As in our case, this patient had pre-existing diabetes (Non-Insulin-Dependent) with possible ocular compromise. However, unlike our patient, he also had some evidence of hemolytic anemia.$^{2}$ 
There is a possibility of OA even at therapeutic doses of dapsone, but the mechanism is not well explained, which is also true in cases of dapsone overdose. The risk of developing OA could be increased by other predisposing factors that cause optic ischemia, including old-age, HTN, DM, dyslipidemia and coagulopathy. ${ }^{8}$ It is advised to have regular ophthalmic checkups prior to dapsone therapy; and we should be more vigilant in case of patients with added risk factors.

\section{References}

1 Wozel VEG. Innovative use of dapsone. Dermatol Clin, 2010; 28: 599-610.

2 Wozel, G., Blasum, C. Dapsone in dermatology and beyond. Arch Dermatol Res 306, 103-124 (2014). https://doi.org/10.1007/s00403-013-1409-7

3 Homeida M, Babikr A, Daneshmend TK. Dapsone-induced optic atrophy and motor neuropathy. Br Med J, 1980; 281(6249): 1180, [Internet]. Available from: https://www.ncbi.nlm.nih.gov/pmc/articles/PMC1714486/.

4 Stanfield JP. A case of acute poisoning with dapsone. J Trop Med Hyg, 1963; 66: 292-295.

5 Cooke TJL. Dapsone poisoning. Med J Aust, 1970; 1(23): 1158-1159, [Internet]. Available from: https://onlin elibrary.wiley.com/doi/abs/10.5694/j.1326-5377.1970.tb84486.x.

6 Davies R. Fatal poisoning with udolac (diaminodiphenylsulphone). Lancet, 1950; 255(6611): 905-906, [Internet]. Available from: http://www.thelancet.com/article/S0140673650907367/fulltext.

7 Hye Park K, Kim H, Chongseo Lee C, Chul Cha K, Min Park S, Jin Ji H et al. Dapsone intoxication: clinical course and characteristics. Clin Toxicol, 2010; 48(6): 516-521, [Internet]. Available from: http://www.tandfon line.com/doi/full/10.3109/15563650.2010.490534.

8 Farunfelder FTGJ. In: Farunfelder FTGJ (ed.), Drug-induced ocular side effects. 4th ed, Baltimore: Williams and Wiklins 1996; pp. 57-58.

9 Chakrabarti M, Suresh PN, Namperumalsamy P. Bilateral macular infarction due to diaminodiphenyl sulfone (4,4’ DDS) toxicity. Retina, 1999; 19(1): 83-84, [Internet]. Available from: http://www.ncbi.nlm.nih.gov/pubm ed/10048382.

10 Chalioulias K, Mayer E, Darvay A, Antcliff R. Anterior ischaemic optic neuropathy associated with dapsone, vol. 20, Eye. Nature Publishing Group 2006; pp. 943-945, [Internet]. Available from: https://www.nature.com /articles/6702050.

11 Biswas SK. Chemotherapy of leprosy. J Indian Med Assoc, 2004; 102: 695-698.

12 Sapkota BR, Shrestha K, Pandey B, Walker SL. A retrospective study of the effect of modified multi-drug therapy in Nepali leprosy patients following the development of adverse effects due to dapsone. Lepr Rev, 2008; 79(4): $425-428$.

13 Kenner DJ, Holt K, Agnello R, Chester GH. Permanent retinal damage following massive dapsone overdose. $\mathrm{Br}$ J Ophthalmol, 1980; 64(10): 741-744, [Internet]. Available from: https://europepmc.org/articles/PMC104380 8.

14 Cream JJ, Scott GL. Anaemia in dermatitis herpetiformis: the role of dapsone-induced haemolysis and malabsorption. Br J Dermatol, 1970; 82(4): 333-342, [Internet]. Available from: https://pubmed.ncbi.nlm.ni h.gov/5441766/.

15 McCulley TJ, Lam BL, Feuer WJ. A comparison of risk factors for postoperative and spontaneous nonarteritic anterior ischemic optic neuropathy. J. Neurooncol, 2005; 25: 22-24.

16 Tsai RK, Liu YT, Su MY. Risk factors of non-arteritic anterior ischemic optic neuropathy (NAION): ocular or systemic. Kaohsiung J Med Sci, 1998; 14(4): 221-225, Available from: https://pubmed.ncbi.nlm.nih.gov/9589 $616 /$.

17 Hayreh SS. Role of nocturnal arterial hypotension in the development of ocular manifestations of systemic arterial hypertension. Curr Opin Ophthalmol, 1999; 10(6): 474-482, [Internet]. Available from: https://pubme d.ncbi.nlm.nih.gov/10662254/.

18 Bajracharya K, Gautam P, Yadav SK, Shrestha N. Epidemiology and causes of optic atrophy in general outpatient Department of Lumbini Eye Institute. J Univers Coll Med Sci, 2016; 3(2): 26-29.

19 Leonard JN, Tucker WFG, Fry L, Marsh RJ, Ford S. Dapsone and the retina. Lancet, 1982; 319: 453, [Internet]. Available from: https://linkinghub.elsevier.com/retrieve/pii/S0140673682916580. 